Ohmic H-mode and confinement in TCV

This article has been downloaded from IOPscience. Please scroll down to see the full text article. 1995 Plasma Phys. Control. Fusion 37 A215

(http://iopscience.iop.org/0741-3335/37/11A/014)

View the table of contents for this issue, or go to the journal homepage for more

Download details:

IP Address: 128.178.125.142

The article was downloaded on 18/02/2013 at 08:53

Please note that terms and conditions apply. 


\section{Ohmic H-mode and confinement in TCV}

J.-M. Moret, M. Anton, S. Barry, R. Behn, G. Besson, F. Bühlmann,

A. Burri, R. Chavan, M. Corboz, C. Deschenaux, M.J. Dutch, B.P. Duval, D. Fasel, A. Favre, S. Franke, A. Hirt, F. Hofmann, C. Hollenstein, P.-F. Isoz, B. Joye, J.B. Lister, X. Llobet, J.-C. Magnin, P. Mandrin, B. Marletaz, P. Marmillod, Y. Martin, J.-M. Mayor, J. Moravec*, C. Nieswand, P.J. Paris, A. Perez, Z.A. Pietrzyk, V. Piffl*, R.A. Pitts, A. Pochelon, O. Sauter, W. van Toledo, G. Tonetti, M.Q. Tran, F. Troyon, D.J. Ward, H. Weisen

Centre de Recherches en Physique des Plasmas

École Polytechnique Fédérale de Lausanne

Association Euratom - Confédération Suisse

CH-1015, Lausanne, Switzerland

* Institute of Plasma Physics

Czech Academy of Science

Za Slovankou 3, 18200 Praha 8, Czech Republic

Abstract: The unique flexibility of TCV for the creation of a wide variety of plasma shapes has been exploited to address some aspects of tokamak physics for which the shape may play an important role. The electron energy confinement time in limited ohmic L-mode plasmas whose elongation and triangularity have been varied $(\kappa=1.3-1.9$, $\delta=0.1-0.7$ ) has been observed to improve with elongation as $\kappa^{0.5}$ but to degrade with triangularity as $(1-0.8 \delta)$, for fixed safety factor. Ohmic $\mathrm{H}$-modes have been obtained in several diverted and limited configurations, with some of the diverted discharges featuring large ELMs whose effects on the global confinement have been quantified. These effects depend on the configuration: in double null (DN) equilibria, a single ELM expels on average $2 \%, 6 \%$ and $2.5 \%$ of the particle, impurity and thermal energy content respectively, whilst in single null (SN) configurations, the corresponding numbers are $3.5 \%, 7 \%$ and $9 \%$, indicative of larger ELM effects. The presence or absence of large ELMs in DN discharges has been actively controlled in a single discharge by alternately forcing one or other of the two X-points to lie on the separatrix, permitting stationary density and impurity content $\left(\mathrm{Z}_{\mathrm{eff}} \approx 1.6\right)$ in long $\mathrm{H}$-modes $(1.5 \mathrm{~s})$. 


\section{Introduction}

The principal goal of TCV (Tokamak à Configuration Variable) is to investigate effects of the plasma shape, in particular high elongation, on tokamak physics and performance. The shape variability of TCV can cover high elongations, $k \leq 3$, and high positive or negative triangularity (with independent control of the top and bottom triangularity). A wide variety of open diverted configurations are possible, including single null (SN) or double null (DN) equilibria with a large variation in X-point position, divertor leg geometry and strike zone location and extension. In order that these different shapes may be created without any hardware modification, the TCV design incorporates a number of unusual features. The vacuum vessel has a nearly rectangular cross-section with a height-to-width ratio of 3 and is continuously welded. Its low toroidal resistance $(55 \mu \Omega)$ allows passive stabilisation of vertical plasma movement at high elongation. Plasma shaping is accomplished by a set of 16 independently controlled poloidal coils, arranged in two vertical stacks of 8 on each side of the vessel. Other constructional parameters are major radius, $R=0.89 \mathrm{~m}$, minor radius, $a \leq 0.25 \mathrm{~m}$, toroidal magnetic field, $\mathrm{B}_{\mathrm{T}} \leq 1.43 \mathrm{~T}$ and maximum plasma current at full elongation, $\mathrm{I}_{\mathrm{p}}>1 \mathrm{MA}$.

From the beginning of physics operation in June 1993, a large effort has been devoted to the establishment and optimisation of the extremely versatile shape control system required to exploit the potential of TCV. The combination of the outer coil power supply bandwidth and the shielding effect of the vacuum vessel limits the maximum vertical instability growth rate, $\gamma$, and hence the maximum plasma elongation that can be stabilised. To date, the highest elongation achieved is $\mathrm{K}=2.05$ at $I_{p}=800 \mathrm{kA}$ and, in a separate discharge, a maximum value of $\gamma=1000 \mathrm{~s}^{-1}$ has been obtained. To permit stabilisation of the highest elongations and hence allow TCV to reach its full design specification, internal coils have been installed inside the vessel and a dedicated fast power supply is now being commissioned for operation later in 1995. Installation of a 4.5 MW ECRH system is also underway to permit further improvement in plasma performance.

This paper addresses a number of physics issues for which the study of the influence of plasma shape is important. First results will be presented in each case. The first subject, discussed in Section 2, treats the influence of shape on global energy confinement, for which the present database is rather sparse, comprising mainly extrapolations of results from different machines. As a second topic, the ohmic H-mode, which has been achieved in virtually all configurations in TCV will be presented in Sections 3 and 4. Section 3 gives a phenomenological description of the H-mode, whilst Section 4 describes the impact of ELMs on the global confinement in many configurations. The discussion of Section 5 will show how fine shape adjustments can control the ELM occurrence. The last, Section 6 , explores the present TCV operational domain, describing results of measurements and modelling of the vertical instability growth rate in several configurations. 


\section{Influence of plasma shape on confinement}

Given the wide variety of possible TCV equilibria, a first step in the approach to quantifying the influence of plasma shape on confinement properties has been to concentrate on the study of a few specific shapes. Since elongation and triangularity are two of the most important shape parameters, both an elongation scan at fixed triangularity and a scan in triangularity, $\delta$, at fixed elongation have been performed. Configurations with values of $\mathrm{K}=1.3,1.5,1.7$ and 1.9 at a fixed $\delta=0.3$ and $\delta=0.1,0.3,0.5$ and 0.7 at a fixed

Elongation scan

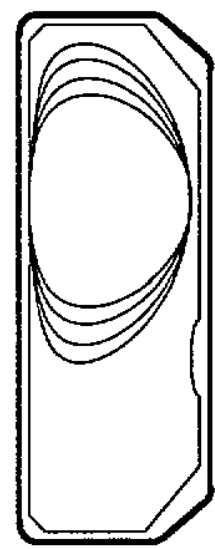

Triangularity scan

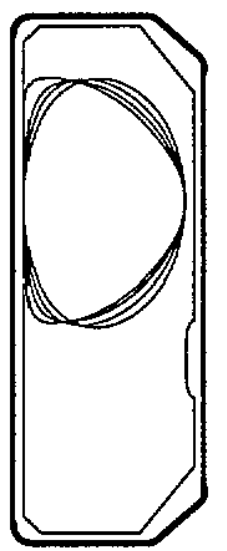

Fig. I The elongation and triangularity scan used for the confinement study. $\kappa=1.5$ have been generated. Analytic shape contour coordinates were used: $R=R_{0}+a \cos (\theta+\delta \sin \theta)$ and $Z=Z_{0}+$ a $K \sin \theta$ where $\left(R_{0}, Z_{0}\right)$ refers to the plasma centre, $a$ is the minor radius and $\theta$ the poloidal angle [1]. Figure 1 illustrates these 7 shapes. It is impossible to maintain constant plasma current with such large shape modifications, due both to important variations in the edge safety factor and because the smallest configurations cannot accommodate large currents and the largest cannot be produced at low currents. A current scan has thus been performed in each configuration in order to encompass a variation of the edge safety factor $\mathrm{q}_{\mathrm{a}}$ from 3 to 5 , except at the highest elongation, for which the low currents corresponding to values of $\mathrm{q}_{\mathrm{a}}>4$ lead to a high degree of vertical instability which cannot at present be controlled in TCV. These current scans are necessary to separate any influence of the shape on the confinement from an intrinsic dependence on the plasma current. Each discharge was performed at the same line averaged density, $8 \times 10^{19} \mathrm{~m}^{-3}$, so that any density dependence of the confinement need not to be accounted for. For each configuration, the plasma remained in L-mode and was limited by the graphite inner wall protection tiles. All data points were taken during stationary conditions, so that time derivatives of magnetic and thermal energy can be ignored during the analysis.

Since a measurement of the total radiated power, $P_{\text {rad }}$, is not yet available, the confinement properties of these plasmas are quantified simply by the electron energy confinement time, $\tau_{\mathrm{Ee}}=\mathrm{W}_{\mathrm{e}} / \mathrm{P}_{\mathrm{oh}}$, where $\mathrm{P}_{\mathrm{oh}}$ is the ohmic input power. The total electron energy, $W_{e}$, is obtained by volume integration of Thomson scattering measurements at up to 10 spatial positions. The electron energy confinement time is plotted in figure 2 as a function of the inverse safety factor, $1 / \mathrm{q}_{\mathrm{a}}$, for all configurations. In all cases a decrease of $\tau_{E e}$ is observed when $I_{p}$ is increased, compatible with a $q_{a}{ }^{0.3}$ scaling and somewhat 
weaker than in the neo-AIcator law. As shown by the decreased value of the ratio of the central value of the density to its volume average, the density profile broadens at high $\mathrm{I}_{\mathrm{p}}$. Thus, although the line average density remains constant, some residual dependency on the central density may also explain part or all of this variation of $\tau_{\mathrm{Ee}}$.
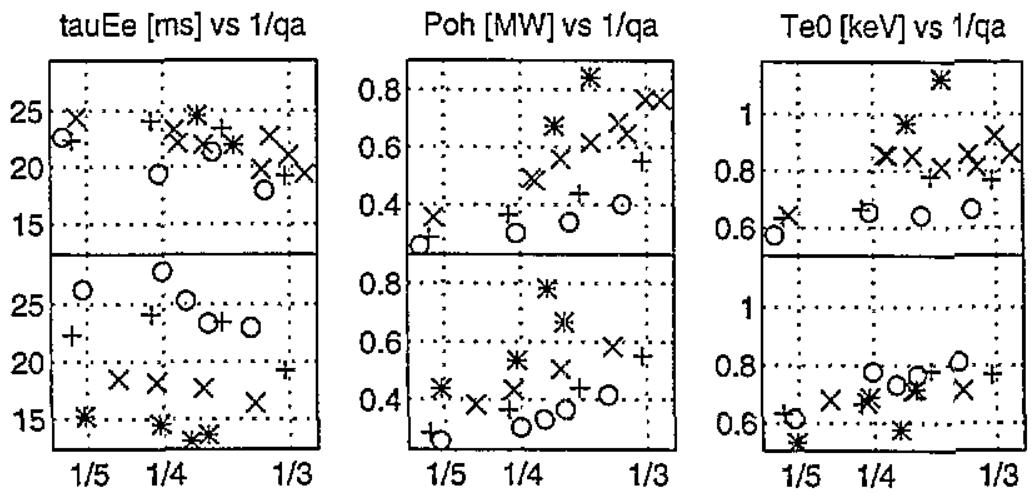

tauEe [ms] vs Ip [MA]
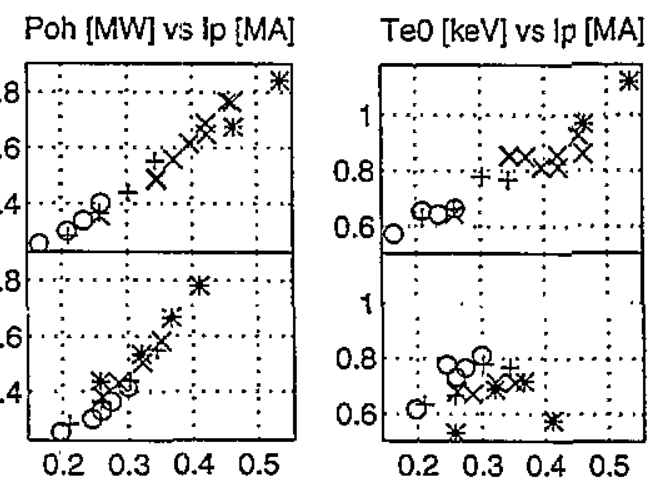

Fig. 2 Electron energy confinement time, ohmic power and central electron temperature plotted as a function of the inverse safety factor and of the plasma current. The following symbols are used:

$\begin{array}{llcccc} & & 0 & + & \times & * \\ \text { top half of each figure: elongation scan } & \kappa= & 1.3 & 1.5 & 1.7 & 1.9 \\ \text { bottom half of each figure: iriangularity scan } & \delta= & 0.1 & 0.3 & 0.5 & 0.7\end{array}$

Turning specifically to the elongation scan, the first observation shown in figure 2 is a large increase in the central electron temperature, $T_{e}(0)$, rising from $0.6 \mathrm{keV}$ at the lowest current and elongation to $1.2 \mathrm{keV}$ for the highest current and elongation. This leads to a relative increase in $\mathrm{W}_{\mathrm{e}}$ larger than that of the plasma volume. In addition, the increase in the electric field necessary to drive the current (roughly proportional to $\left.\left(\kappa^{2}+1\right) / 2\right)$ is compensated by a decrease in the plasma resistivity, resulting in a loop voltage, $V_{\text {loop }}$, that, at a given value of $q_{a}$, is the same for all elongations. Combining these effects gives a $\tau_{E e}$ which improves with elongation at a fixed value of $q_{a}$; this dependence may be described by a power law, $k^{0.5 \pm 0.1}$. The ratio of the total energy confinement time predicted by the ITER89-1? scaling law and the measured electron energy confinement time ranges from 0.6 to 1.2 , which may indicate that the parametric 
dependence of ITER scaling is niot incorrect. Extracting the varying parameters relevant to this elongation scan from the ITER89-P law yields a factor $2.5 \mathrm{I}_{\mathrm{p}}^{0.85} \mathrm{~K}^{0.5} \mathrm{P}_{\mathrm{oh}}{ }^{-0.5}$, where the numerical coefficient (for $I_{p}$ in MA and $P_{o h}$ in $M W$ ) is specific to the TCV conditions and is chosen such that this factor is unity when extrapolating to $\kappa=1$. The result is a predicted improvement of the confinement time that can be summarised by $\kappa^{1.2}$ and, as illustrated in figure 3 , is substantially larger than that observed in TCV. The ITER89-P law essentially applies to confinement in presence of additional heating; in ohmic plasmas the intrinsic link between $I_{p}$ and $P_{o h}$ may render the comparison impossible. At high elongation, the increase in both $P_{o h}$ and $T_{e}(0)$ results in an increased impurity concentration, observed on the soft X-ray emissivity [2] and on the intensity of a C-VI line measured by an ultra-soft X-ray spectrometer [3]. The concentration of carbon, the main impurity in TCV, deduced from these measurements is roughly proportional to the ohmic power leading to a ratio $\mathrm{P}_{\mathrm{rad}} / \mathrm{P}_{\text {oh }}$ that should be almost constant. Thus, a more refined estimate of $\tau_{\mathrm{Ee}}$ accounting for the radiated power would give a correction factor that is essentially identical for all elongations and would not fundamentally modify the observed $\kappa$ dependence.

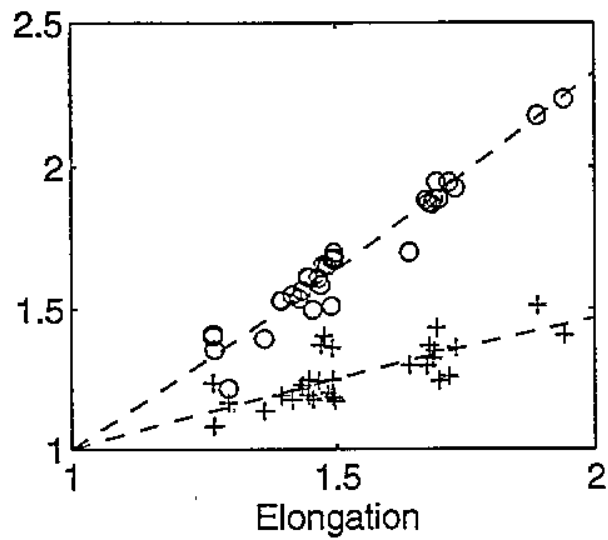

Fig. 3 Improvement-factor of the electron energy confinement time when elongation is increased. Circles are the ITER89-P predictions, crosses the TCV measurements.

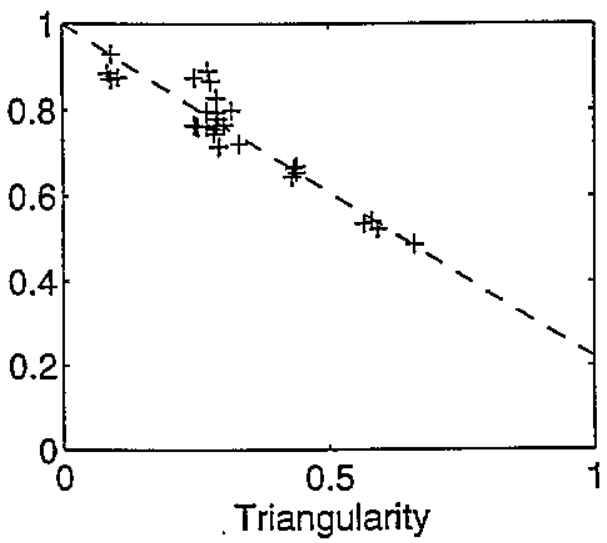

Fig. 4 Degradation factor of the electron energy confinement time when triangularity is increased.

In the triangularity scan, the current must be increased as $\delta$ varies in order to

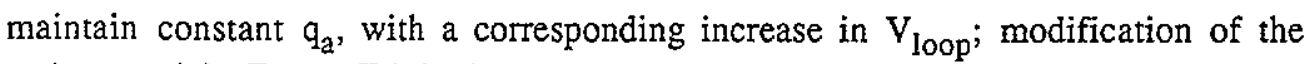
resistance (via $Z_{\text {eff }}$ or $T_{e}$ ) is, in contrast to the elongation scan, only marginal. The relative reduction of the electrical conductivity due to a larger fraction of trapped electrons at high triangularity is computed to be less than $5 \%$ for the highest triangularity and has also only a small influence on $V_{10 o p}$. The combination of these effects leads to a substantial reduction in $\tau_{\mathrm{Ee}}$ of roughly $40 \%$ when ${ }^{\prime}$ passing from an elliptical shape to a triangularity of 0.7 . This reduction cannot be explained by an intrinsic dependence on the plasma current alone; this clearly appears in a plot (figure 2) of $\tau_{E e}$ versus $I_{p}$ in which each triangularity forms a decreasing curve shifted with respect to the others. A small reduction in the impurity concentration is observed 
at fixed plasma current when increasing the triangularity. Thus the ratio $\mathrm{P}_{\mathrm{rad}} / \mathrm{P}_{\text {oh }}$ is decreasing and a confinement time corrected for the radiation loss would display an even larger degradation when the shape is more triangular. Whilst a power law cannot be used, figure 4 shows that for the $\delta$ variation explored in this study, a reduction factor of the form $(1-0.8 \delta)$ approximately describes the present data.

TCV shot \# $5645 \mathrm{SN}$

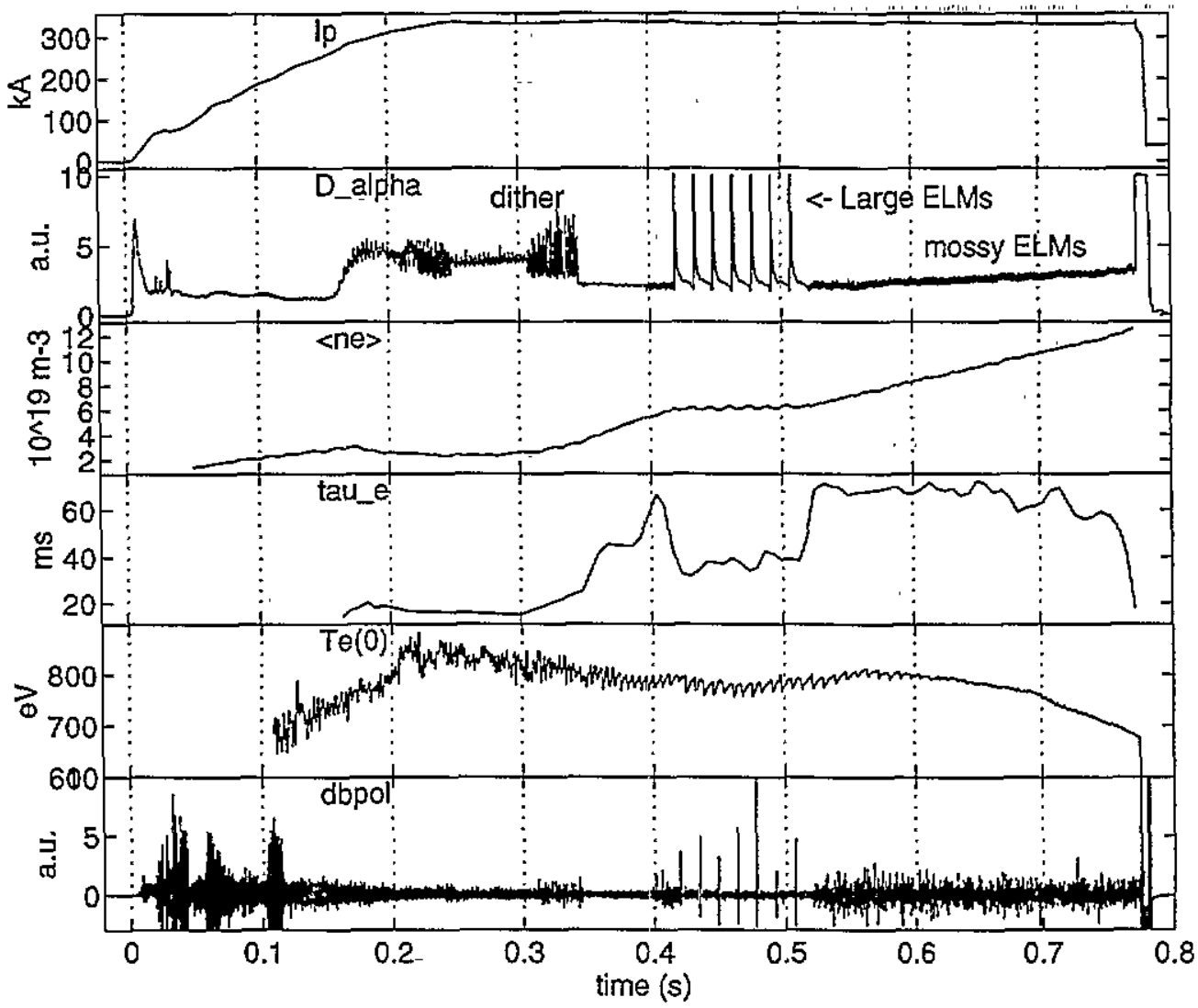

Fig. 5 Typical H-mode time traces. From top to bottom: plasma current, $\mathrm{D}_{\alpha}$ signal, average electron density, energy confinement time, electron temperature from soft X-ray emission, MHD magnetic activity on the high field side midplane.

\section{Ohmic H-mode phenomenology}

Following the first boronisation in TCV, ohmic H-modes were obtained in discharges with a variety of shapes, including SN equilibria with the ion $\nabla B$ drift directed towards the $\mathrm{X}$-point and $\mathrm{DN}$ configurations [4]. Limiter $\mathrm{H}$-modes have also been achieved for $\kappa$ between 1.7 and 1.9 at $\mathrm{I}_{\mathrm{p}}$ ranging from 360 to $600 \mathrm{kA}$, although $\mathrm{H}$ transitions are only possible at $360 \mathrm{kA}$ for values of $\delta \geq 0.25$. The discharge shown in figure 5 illustrates most of the features observed in TCV $\mathrm{H}$-modes. Transitions can be "dithering", with the $D_{\alpha}$ intensity switching between $L$ - and $H$-mode levels and can also feature ELMs with pulses of $D_{\alpha}$ emission well above the L-mode level. In general, 
an initial ELM-free period of $20 \mathrm{~ms}$ or more follows the L-H transition. In the example presented in figure 5, a short period of irregular "mossy" ELMs causing low amplitude fluctuations on the $D_{\alpha}$ signal precedes several large ELMs. The latter significantly reduce the energy confinement time and interrupt the density rise with, in this particular case, the H-phase terminating after a final period of mossy ELMs characterised by MHD activity and $D_{\alpha}$ modulation localised in the divertor region and do not appear to influence the global confinement. The fact that the first large ELM is observed only after an initial ELM-free period during which an edge pressure gradient can build up, suggests that these large ELMs may be related to type I ELMs observed in other devices with additional heating. There is no physics reason why this type of ELM should exist only in the presence of additional heating, provided that the edge confinement is effective enough to bring the pressure gradient there to the ELM instability limit.

Fig 6 SN and DN equilibria used for quantification of the ELM effects.

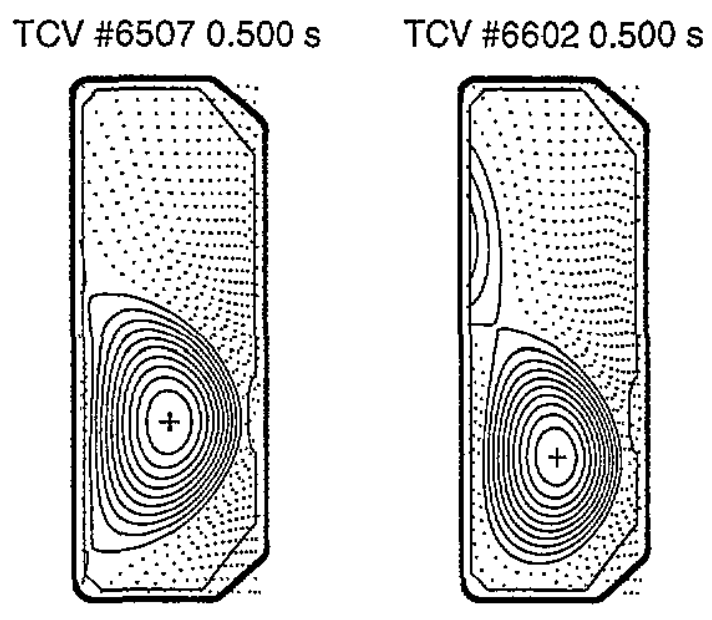

4. Effect of ELMs on the global confinement

The potential for using ELMs as a means of controlling the impurity and $\alpha$-particle content of an $\mathrm{H}$-mode based reactor plasma requires knowledge both of how to control their occurrence and of their effect on global confinement. One method by which the latter may be investigated is to take the time average of particle and energy balance over many ELMs [5]. This approach allows an estimation of the average effect of a single ELM without requiring detailed measurements of relevant parameters for each ELM. In the case of electron particle balance this reads:

$$
<\mathrm{dN} / \mathrm{dt}>/ \mathrm{N}=\mathrm{S} / \mathrm{N}-1 / \tau_{\mathrm{up}}-\mathrm{f}_{\mathrm{ELM}}<\Delta \mathrm{N}_{\mathrm{ELM}}>/ \mathrm{N}
$$

where the LHS is the rate of change of the total electron content averaged over many ELMs, $S$ is a particle source, $\tau_{\text {up }}$ stands for the underlying particle confinement time (that which would prevail in the absence of ELMs), $f_{E L M}$ symbolises the ELM 
repetition rate or frequency and $\left\langle\Delta \mathrm{N}_{\mathrm{ELM}}\right\rangle$ is the average number of particles expelled by a single ELM. Analogous expressions hold for the impurity content, $\mathrm{N}_{\mathrm{Z}}$, and the thermal energy $W$. Only the quantities $\left\langle\mathrm{dN} / \mathrm{d} \backslash \mathbb{N}\right.$ and $f_{\mathrm{ELM}}$ can be directly measured, but the relative number of electrons expelled by each ELM, $\left\langle\Delta N_{E L M}>/ N\right.$, may be evaluated from the slope of $\left\langle\mathrm{dN} / \mathrm{d} t>\mathbb{N}\right.$ as a function of $\mathrm{f}_{\mathrm{ELM}}$.
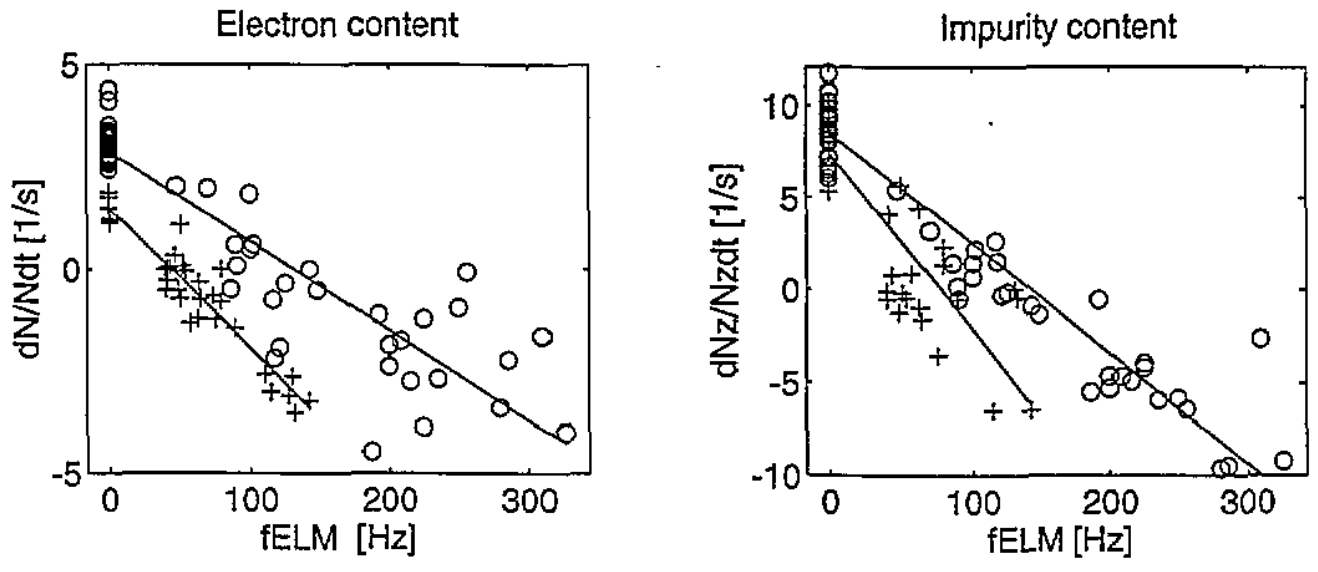

Thermal energy
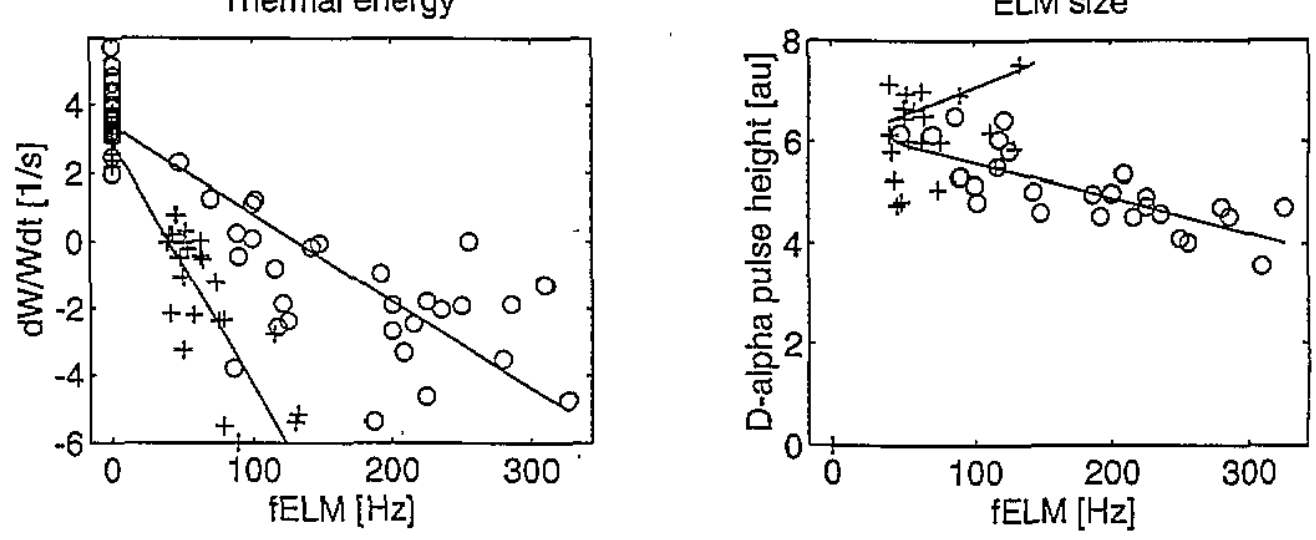

Fig. 7 Relative rate of change of the electron particle, the impurity and the thermal energy content as a function of the ELM frequency for the $S N(+)$ and $D N(0)$ configuration.

Two configurations have been studied in detail, a DN D-shaped configuration with $\kappa=1.7, \delta=0.7$ and $\mathrm{T}_{\mathrm{p}}=390 \mathrm{kA}$ and a SN configuration with the ion drift directed toward the $X$-point and with $\kappa=1.6, \delta=0.3$ and $I_{p}=330 \mathrm{kA}$, both shown in figure 6 . In these experiments the total electron content was measured by a 4-channel interferometer, the impurity content estimated from the soft X-ray emissivity [2] and the total thermal energy derived from the equilibrium reconstruction based only on magnetic measurements. Each experimental trace is divided into time windows of roughly constant ELM frequency in which the relevant quantities and their time derivatives are estimated. The corresponding rates of change are plotted in figures 7 as a function of $f_{E L M}$ for both configurations, together with the $D_{\alpha}$ spike amplitude. The latter varies only slightly over the observable frequency range. The rates of change are nearly linear 
with $f_{E L M}$ up to frequencies of about $300 \mathrm{~Hz}$ and $120 \mathrm{~Hz}$ for the DN and the SN configurations respectively. Above these frequencies small, irregular ELMS and H-I transitions render this type of analysis impracticable. The slopes of the fitted lines indicate that in DN each ELM expels on average $2 \%$ of the electron content, $2.5 \%$ of the thermal energy and $6 \%$ of the impurity content. In SN, the corresponding numbers are $3.5 \%, 7 \%$ and $9 \%$ and are indicative of larger ELM effects in this configuration. Note that this is an estimate of the net loss fraction of particles, since the gross outward flux due to the ELMs may be partly offset by an influx due to ELM-enhanced recycling. In addition, the estimate of the impurity content is based only on a single chord measurement and cannot account for a spatial distribution or a profile change due to the ELMs. The fraction of thermal energy released by the ELMs is observed to be larger than that of the particle content in both configurations. This shows that the expelled energy is larger than the initial thermal energy of the expelled particles and suggests a transient enhancement in transport larger for the heat than for the particles. In DN, stationary conditions are obtained for an ELM frequency of $120 \mathrm{~Hz}$, where the fitted line crosses zero. At this point, the volume average electron density is $\left\langle\mathrm{n}_{\mathrm{e}}\right\rangle \approx$ $8 \times 10^{19} \mathrm{~m}^{-3}$, the effective charge $Z_{\text {eff }} \approx 1.6$ and the energy confinement time is reduced by $\approx 30 \%$ compared with the underlying value in the absence of ELMs. In SN, due to the larger effect of individual ELMs, stationary conditions are obtained at a lower ELM frequency of $50 \mathrm{~Hz}$.

\section{ELM control experiments}

During H-mode DN experiments, the presence of large ELMs has been observed to be correlated with a slight poloidal flux imbalance between the two X-points. The ELMs appear only if the ion $\nabla B$ drift is directed away from the active $\mathrm{X}$-point, defined as that lying on the separatrix. This phenomena has been used to actively control the ELM occurrence [6]. Switching between active upper and lower X-points (figure 8) is achieved simply by modulating the vertical position reference by a few millimetres. The most striking feature in figure 8 is the synchronisation of the ELM-free and ELMy phases with the imposed modulation, in this case $\pm 12 \mathrm{~mm}$. In this way, H-modes of long duration, up to $1.5 \mathrm{~s}$, have been obtained in which a quasi-stationary density, $\left.<\mathrm{n}_{\mathrm{e}}\right\rangle \approx 8 \times 10^{19} \mathrm{~m}^{-3}$, is achieved despite the ELM-free periods. Although higher than its $\mathrm{L}$-mode level, the average impurity concentration also remains stationary at $\mathrm{Z}_{\mathrm{eff}} \approx$ 1.6. This technique has also been used to feedback control the density during $\mathrm{H}$-mode, by appropriately coupling the density error signal to the vertical position demand signal. Under these conditions, the plasma was again observed to toggle between the ELMfree and ELMy states. This is indicative of an hysteresis in the establishment of the conditions required for the ELMs to appear. This observation and the fact that large ELMs have never been seen during the first $20 \mathrm{~ms}$ of an $\mathrm{H}$-mode, suggest that not only the configuration, but also other parameters and their time evolution, such as the build up of an edge pressure gradient, are important in determining the stability domain of occurrence of large ELMs. 


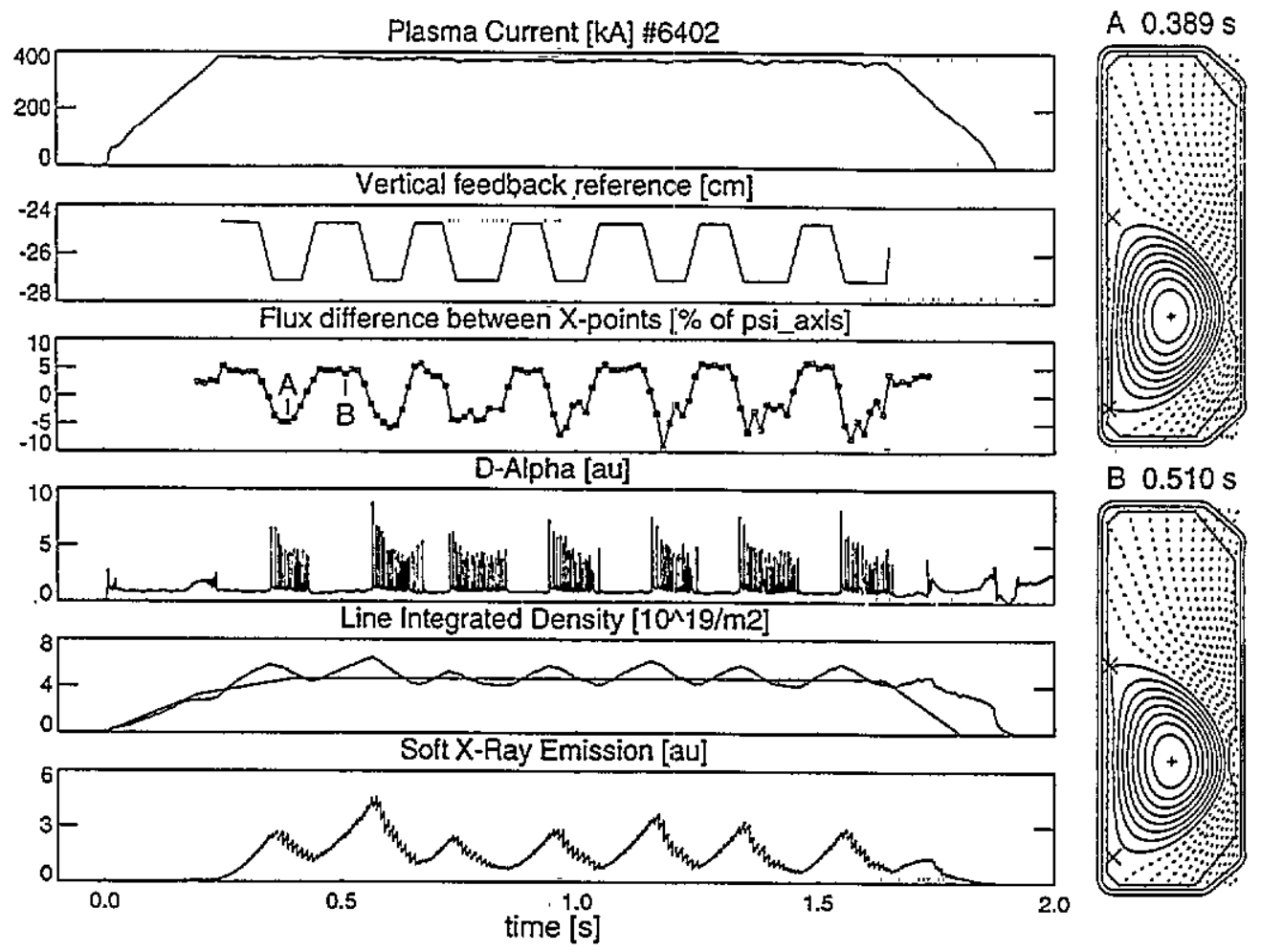

Fig. 8 Active ELM control.

\section{Measurement and modelling of the vertical growth rate}

The very elongated shapes possible in $\mathrm{TCV}$ offer an ideal opportunity to experimentally determine vertical instability growth rates for a variety of plasma shapes and to compare the results with modelled values [7]. A series of discharges has been performed in which both the vertical and radial position feedback loops acting on the outer shaping coils were opened following establishment of the final configuration. The plasma motion during the subsequent vertical displacement disruption was monitored both by the real time observer of the centre of gravity of the current distribution (as described in [1]) and by the equilibrium reconstruction. In each case, a vertical growth rate was extracted from the exponential temporal evolution. Three shape parameters were varied at a fixed plasma current of $250 \mathrm{kA}$ : an elongation scan from $\kappa=1.4$ to 1.75 , a triangularity scan from $\delta=0.1$ to 0.65 and a vertical position scan from the vessel mid-plane, $\mathrm{Z}_{\mathrm{o}}=0 \mathrm{~m}$, to $0.3 \mathrm{~m}$. The measured growth rates are plotted in figure 9 and show that the vertical instability increases rapidly with elongation. The maximum observed value of $\gamma=1000 \mathrm{~s}^{-1}$ is the highest yet measured on TCV. Combined with the time constants of the vessel eigenmode current decay (2.7 to $8.2 \mathrm{~ms}$ ), this value of $\gamma$ corresponds to a stability margin of only 1.26 but is close to the maximum frequency response of the thyristor power supplies. The results demonstrate the beneficial effect of imposing strong triangularity when increasing the elongation, due to a reduction of the field curvature at fixed $\kappa$ when $\delta$ is increased. The 
scan in vertical position indicates the importance in TCV of the proximity of the wall in making the vertical motion less unstable, due to better coupling of the plasma current distribution to the top or bottom vacuum vessel plates. These experimental values have been compared both with the predictions of a rigid displacement model of the current distribution in the presence of the vessel [8] and with calculations of the NOWA-W code [9] which also includes plasma deformability. Both models give good agreement with the experimental observations.

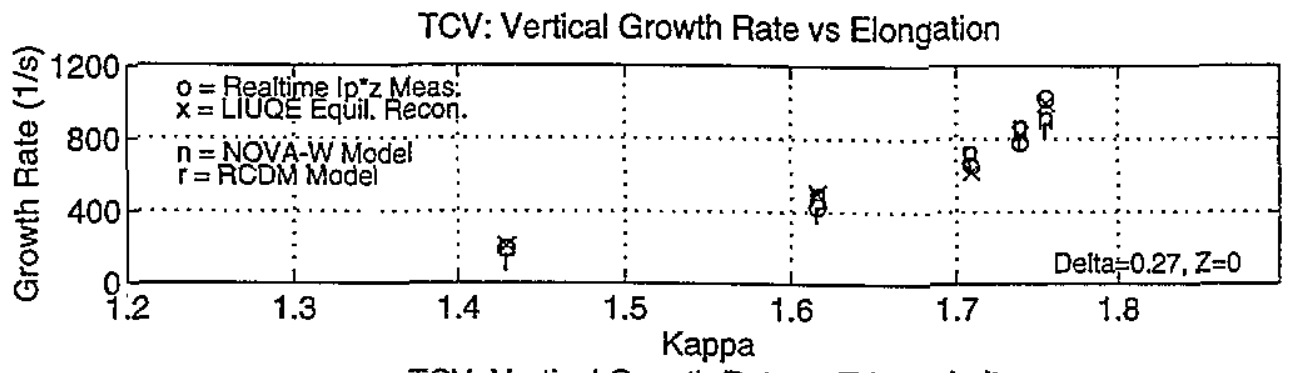

TCV: Vertical Growth Rate vs Triangularity

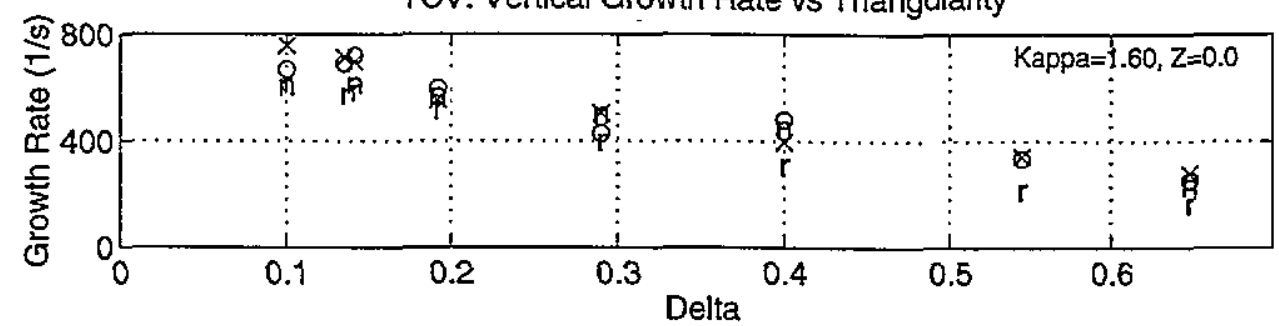

TCV: Vertical Growth Rate vs Initial Vertical Position

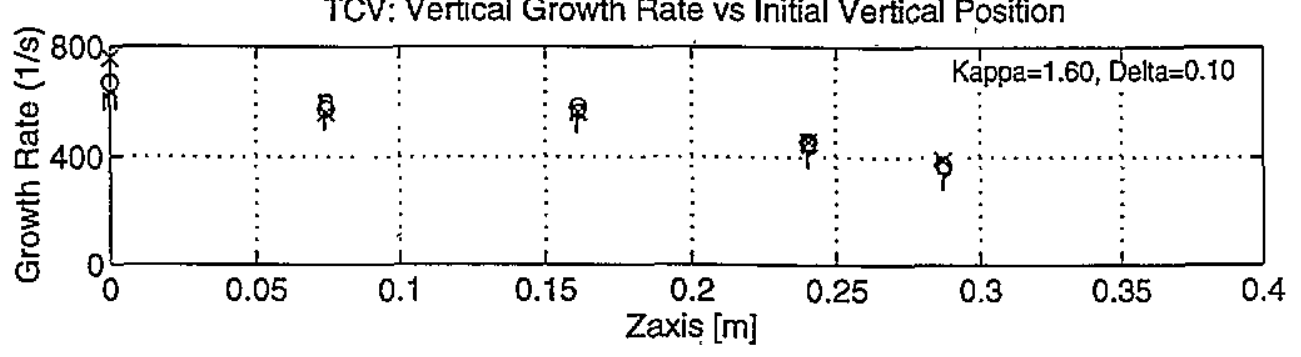

Fig. 9 Measturements and modelling of the vertical instability growth rate for an elongation, triangularity and vertical position scan.

\section{Conclusions}

After two years of operation, TCV has explored a large variety of plasma shapes, demonstrating the unique flexibility of the device and its control systems. Experiments have been performed that address specific aspects of tokamak physics for which the plasma shape plays an important role. The influence of elongation and triangularity on the confinement properties have begun to be explored in L-mode limited configurations. Preliminary results indicate that at a fixed value of the safety factor, the electron energy confinement time improves with the elongation as $\mathrm{k}^{0.5}$, but deteriorates at high triangularity by a factor $(1-0.8 \delta)$ for the equilibria studied. These important results 
need to be confirmed and the database enlarged to encompass a wider experimental parameter range, including additional heating. Together with improved diagnostic capability, this will hopefully permit a deeper insight into the underlying mechanisms governing these observations.

Ohmic H-modes in TCV are possible in all diverted configurations in which the ion $\nabla B$ drift is directed toward the active $X$-point and even in limited discharges. Large ELMs, probably related to type I ELMs seen in other machines with additional heating have been obtained. Their occurrence and the magnitude of their effect on the global confinement can be modified by varying the plasma configuration, especially near the $X$-points: SN equilibria display larger ELMs than DN equilibria and a slight imbalance of the X-points of DN in favour of the ion $\nabla B$ drift allows ELM suppression. This opens up the possibility of controlling the evolution of $\mathrm{H}$-mode plasma parameters by appropriate tuning of discharge confinement properties through shape modifications.

Acknowledgements. It is a pleasure to acknowledge the entire TCV technical team for their continuous effort during the construction and the operation of the machine. This work was partially supported by the Fonds National Suisse de la Recherche Scientifique.

\section{References}

[1] Hofmann F, Dutch M J, Moret J-M, 1995, Plasma shape control in TCV using $M G A M S$, this conference.

[2] Weisen H, Pasini D, Weller A et al., 1991, Rev. Sci. Instrum. 621531.

[3] Weisen H, Piffl V, Krejci A, Moravec J, Raus J, Dutch M J, Paris P J, 1995, Ultrasoft $X$-ray spectroscopy using multilayer mirrors on $T C V$, this conference.

[4] Hofmann F, Lister J B, Anton M, Barry S, Behn R, Bernel S, Besson G et al, 1994, Plasma Phys. Control. Fusion 36 B277.

[5] Weisen H, Dutch M J, Pochelon A, Hirt A., Pitts R A; Hofmann F, Anton M et al., 1995, Edge localised modes in the TCV Tokamak, this conference.

[6] Dutch MJ, Hofmann F, Duval BP, Hirt A, Joye B, Lister JB, Martin Yet al., 1995, Nucl. Fusion 35650.

[7] Dutch M J, Hofmann F, Sauter O, Ward D J, Anton M, Moret J-M, 1995, Comparison of experimental and theoretical growth rates of the vertical instability in $T C V$, this conference.

[8] Lister I B, 1995, A rigid displacement model for vertical motion in TCV, Lausanne report LRP 526/95.

[9] Ward D J, Jardin S C, Cheng C Z, 1993, J. Comput. Phys.104 221. 